\title{
GREDENGES AND SUSPENDED JUDGMENTS AS TRANSITIONAL ATTITUDES
}

\author{
Julia Staffel \\ University of Colorado Boulder \\ (forthcoming in Philosophical Issues, penultimate version)
}

\section{Introduction}

In this paper, I want to highlight an interesting difference between belief on the one hand, and suspended judgment and credence on the other hand. This difference is the following: credences and suspended judgments are suitable to serve as transitional as well as terminal attitudes in our reasoning, whereas beliefs are only appropriate as terminal attitudes. The notion of a transitional attitude is not an established one in the literature, but I argue that introducing it helps us better understand the different roles suspended judgments and credences can play in our reasoning. Transitional and terminal attitudes have interestingly different descriptive and normative properties. I also compare my account of transitional attitudes to other inquiry-guiding attitudes that have recently been characterized in the literature and explain why they are different. I will begin by describing a variety of cases that illustrate and motivate the distinction between transitional and terminal attitudes in reasoning, and then move on to discussing its significance.

\section{Motivating Cases}

When we try to settle a question, or make a decision, the process of inquiry we go through can be roughly described as involving two central components. One component is the collection of relevant information. For example, if I want to decide where to go on vacation, I need to do some research on what different options might be, how expensive they are, and so on. In scientific inquiry, we similarly collect empirical information, guided by strict protocols in order to ensure that our evidence is of the right kind. A second component is the evaluation of this information, in order to determine how it bears on the question or decision at hand. For simple questions and decisions, this process is quite trivial, and it will often be executed by our minds entirely automatically, without our noticing the reasoning processes that are being executed "under the hood." However, for more complex cases that require careful, drawn out deliberation, or the evaluation of complex and varied evidence, the second component can be very time- and attentionconsuming. To illustrate this distinction, and to provide a basis for further discussion, I'll now present two cases, each of which has two variants.

\section{(1) Detective Fletcher, conclusive:}

Manny has committed a murder and tries to frame Fred for it. Detective Fletcher, upon initially inspecting the evidence, responds as Manny has planned, and becomes 90\% confident that Fred committed the murder. However, as she evaluates the evidence more carefully, she discovers incongruencies that ultimately lead her to conclude that Fred was framed, so she reduces her confidence that Fred is the murderer to $2 \%$. She also comes to believe that Fred didn't do it. 


\section{(2) Detective Fletcher, inconclusive:}

Manny has committed a murder and tries to frame Fred for it. Detective Fletcher, upon initially inspecting the evidence, responds as Manny has planned, and becomes 90\% confident that Fred committed the murder. However, as she evaluates the evidence more carefully, she discovers incongruencies that ultimately lead her to wonder whether Fred was framed. She is $50 \%$ confident at this point that Fred was framed. She knows that if she goes back to the murder scene, she can verify one way or the other what happened, because she now knows what she failed to attend to the first time around. She returns to the murder scene and finds the piece of evidence she needs to confirm that Fred was framed. She's now $\%$ confident that Fred is the murderer, and she also comes to believe that he didn't do it.

\section{(3) Logic Student, uninterrupted:}

Una is a beginning logic student. She has a list of formulas, and she is asked to determine whether each formula is a tautology or not. She reads the first formula (which is a tautology). Her credence that it is a tautology is $50 \%$. She then writes out the truth table and becomes $80 \%$ confident that it is a tautology. To check, she also produces a natural deduction style proof. As a result, she becomes (virtually) certain that the formula is a tautology.

\section{(4) Logic Student, interrupted:}

Una is a beginning logic student. She has a list of formulas, and she is asked to determine whether each formula is a tautology or not. She reads the first formula (which is a tautology). Her credence that it is a tautology is $50 \%$. She then writes out the truth table and becomes $80 \%$ confident that it is a tautology. Because she is not sure that she did the truth table correctly, she starts writing out a natural deduction style proof, but then she realizes that she has run out of time. She quickly writes "tautology" as her answer on the test sheet.

In each of these four cases, a person is engaged in some kind of inquiry. Detective Fletcher is engaged in a posteriori inquiry into the question of who committed the murder. Una is inquiring an a priori matter, namely whether the formula on her list is a tautology. If we want to distinguish the different phases of their inquiry - the evidence collection phase and the evidence evaluation phase - the most natural way of doing so treats the cases as follows: In (1), Detective Fletcher has already collected all of her evidence. The vignette describes an extended phase of evaluating the evidence and figuring out what it shows. Fletcher transitions from being initially misled by Manny's framing attempt, to then realizing the true impact of the evidence. Fletcher concludes her reasoning by forming a low credence that Fred was the murderer, as well as a corresponding belief that he didn't do it. Case (2) differs in that the first phase of deliberation concludes with a middling credence and suspended judgment about whether Fred was framed. There is then another phase of evidence collection, which is succeeded by a final, (very brief) deliberation phase that results in the same conclusion as in (1). Notice that in both cases, the evidence collection and deliberation phases are never interrupted. Fletcher gets to conclude every phase in both versions of the case. 
In the logic cases, there is no obvious phase of evidence gathering, since logic is a matter of a priori inquiry. However, the student must of course be familiar with the relevant logical concepts in order to even be in a position to properly formulate the question of whether the formula is a tautology, and to reason her way to an answer. On a natural understanding of what is happening in (3) and (4), both vignettes characterize the phase in which the agent is evaluating relevant evidence in order to determine how it bears on the question they are trying to answer. ${ }^{1}$ The main difference between the cases is that in (3), but not in (4), Una gets to finish her deliberation in the way she has planned. In (4), her deliberation is interrupted, and she decides to answer the question (for the purposes of her logic test, at least), based on the $80 \%$ confidence she has from doing the truth table.

Before I move on to explaining the relevance of these examples, and of the distinct stages of inquiry in the next section, I will add some additional stipulations about these cases, to make sure we're on the same page. Readers who think the cases given don't satisfy these stipulations are invited to tweak them as needed. The first stipulation is that the agents in the examples are rational. By this I mean two things: first, the conclusions the agents reach at the end of each phase of inquiry (except in the fourth case, where Una doesn't get to finish her deliberation) are the ones supported by their evidence. Secondly, the agents' reasoning is not in any way defective. Their attitudes evolve as they rationally ought to as the agents' deliberations progress. In technical epistemological language, this means that in cases (1), (2), and (3), the attitudes reached by the agents at the end of each deliberation phase are both propositionally rational or justified (because they are supported by their evidence), and they are also doxastically justified (because they are based on the agents' evidence via proper reasoning). ${ }^{2}$ With our cases, and our distinction between two phases of inquiry in place, we're now in a position to look more closely at the roles different doxastic attitudes are playing in inquiry. This will be the subject of the next section.

\section{Gredences and Suspension as Transitional and Terminal Attitudes}

In a series of insightful papers, Jane Friedman has recently explored the connections between inquiry and different doxastic attitudes, specifically belief (both outright and graded) and suspension of judgment. She argues that outright, or full belief is an inquiry-stopping attitude, and that having a full belief about the answer to some question means that one has a settled opinion about this matter. She argues for the normative claim that there is an incoherence in both believing a complete answer to a question, and also inquiring into that question at the same time (Friedman 2019). Belief, being a settling attitude, is contrasted by her with inquiring attitudes, the central one being suspension of judgment. Other inquiring attitudes that involve suspension are wondering, hoping, being curious about, and so on. Friedman (2017, see also 2013a, b) argues that there is a rational prohibition against believing some claim $p$ and adopting an inquiring attitude towards the question of whether $p$. Such a combination of attitudes is incoherent. Regarding degrees of belief,

\footnotetext{
${ }^{1}$ Below, I discuss the alternative view according to which the reasoning produces new evidence.

2 This distinction was first introduced by Firth (1978). For some recent discussion, see e.g. Turri (2010), Silva (2015) and Dogramaci (2018).
} 
Friedman argues that they should not be seen as settling attitudes that terminate inquiry into some question. She argues for this partly by showing that any degree of belief in some claim $p$ is in principle compatible with suspending judgment about whether $p$. However, it doesn't seem appropriate to conclude from this that degrees of belief are inquiring attitudes in Friedman's sense, since there is no incoherence in both believing that $p$ and having a high degree of belief in $p$. Friedman's distinction between inquiring and settling attitudes thus best applies to attitudes that don't encode uncertainty, such as belief, knowledge and suspension (where the former two don't have probabilistic contents, such as "probably p").

I will rely on Friedman's view to serve as the backdrop for my argument that it is beneficial to make some finer-grained distinctions between different roles doxastic attitudes can play in inquiry. My claims about these distinctions are independent of Friedman's views on belief, so readers who disagree with Friedman are free to substitute their own views of belief into the framework I am about to propose. In section one, I distinguished between two components of inquiry: the evidence-gathering component, and the deliberation component. I am going to focus on the agent's attitudes during deliberation in what follows, and I will assume that the agent is not acquiring new evidence during this phase. Rather, they are just focused on evaluating the significance of the evidence they have for the question they are inquiring into. This assumption will help simplify my discussion, but nothing ultimately hangs on it. To characterize the different roles attitudes can play in reasoning, I suggest that we distinguish between transitional attitudes and terminal attitudes, which I will now describe in turn.

Terminal attitudes are the attitudes we reach when we have finished a deliberation or reasoning process. Barring unusual troubles in evaluating the evidence, an agent usually finishes their deliberation at some point, when they have established (or at least think they have established) the significance of their evidence for the question at hand. All of the attitudes previously discussed - degrees of belief, outright belief, and suspended judgment - are appropriate as terminal attitudes of deliberation processes. This is not in any way surprising, since any of these attitudes might be the ones that are either supported by the agent's evidence, or that are at least seemingly supported (if the agent reasoned badly). Hence, while not all of these doxastic attitudes are appropriate as terminal attitudes of inquiry - only belief can play this role, if we follow Friedman on this - they are all appropriate terminal attitudes of deliberation. This is, again, not shocking, given that some phases of deliberation lead to settling the question being asked, and some don't. We can identify terminal attitudes in our examples as follows: in case (1), Detective Fletcher concludes by believing and assigning high credence that Fred was framed. In case (2) Fletcher determines that her initial evidence is not decisive, and later she determines that the additional evidence she gathers is decisive. In case (3) Una concludes that the formula is a tautology based on the truth table and the natural deduction style proof. Case (4) differs in that Una does not get to conclude her deliberation. Since she runs out of time on her test, she is forced to answer the question based on her reasoning up to that point. (Although she may well sit down later and confirm with the natural deduction style proof that she got the right answer.)

Having identified the terminal attitudes in our examples, it is easy to see that this category leaves out some of the agents' attitudes. This is where the category of transitional attitudes fits in. 
Transitional attitudes are the attitudes that the agent forms at the start of and during their deliberation, before they reach the terminal attitudes that conclude the relevant deliberation phase. Both degrees of belief and suspended judgments are suitable transitional attitudes, whereas beliefs, due to their question-settling nature, are not (again, assuming we accept Friedman's view of belief). As Friedman observes, it is appropriate to suspend judgment on whether $p$ up until one's inquiry delivers a (complete) answer. Similarly for credences.

The difference between credences that function as transitional attitudes and credences that function as terminal attitudes of reasoning is easier to notice than the same difference between suspended judgments, because agents often change their credences through the course of their deliberation. We can see this in our examples: Detective Fletcher is initially fooled by Manny's attempt to frame Fred, which results in a high transitional credence that Fred committed the murder. But once she thinks more about the evidence, she notices that her first impression doesn't survive additional scrutiny, and hence she lowers her credence that Fred did it (this could happen gradually or in jumps, depending on how we fill in the case). In Una's case, she begins with a 50\% credence that the formula is a tautology, then raises it to $80 \%$ after her first go at coming up with a truth table, and only reaches her terminal credence of 1 (or close to 1) upon checking her initial result. By contrast, if we just characterize the agents' transitional attitudes as suspensions, and we don't pay attention to their credences, we can easily miss how much the agent's attitudes evolve in the course of their deliberations. ${ }^{3}$ Generalizing from these observations, we can characterize transitional attitudes as attitudes that agents hold towards the answers to specific questions they consider to be open, and that they adopt before they have concluded their reasoning about how their evidence bears on these questions. ${ }^{4}$

One important thing to notice here is that not every doxastic attitude the agent forms during their deliberation qualifies as a transitional attitude in the above sense. Some of the attitudes the agent forms while deliberating are terminal attitudes, but they are not terminal attitudes towards the claims the agent is ultimately interested in establishing. An example will help clarify this point: suppose Detective Fletcher (in scenario 1) is thinking about what her evidence supports

\footnotetext{
3 Miracchi (forthcoming) actually distinguishes four different ways in which agents may suspend judgment. She differentiates between inquiry-closing suspension, and suspension that motivates further inquiry, both of which can be adopted deliberately or automatically. Her distinctions don't line up with the ones I propose here, but they can easily be combined with each other once we distinguish the two components of inquiry.

${ }^{4}$ When an agent considers a question to be open, they may or may not be interested in finding the answer to the question. For example, I currently consider the question "What is the largest prime under 1000?" to be open for me, but I have no interest in finding out the answer, even though I could do so by spending some time deliberating. The above characterization of a transitional attitude is meant to capture attitudes towards answers to questions that agents consider open, and that they may or may not be interested in settling by reflecting on how their evidence bears on them. Hence, my credence in the claim "The largest prime under 1000 is 997" would count as a transitional attitude on this definition, even though I am not currently trying to figure out if this is the correct answer. Alternatively, we could reserve the notion of a transitional attitude for attitudes towards answers to questions we are actively deliberating about. Then my attitude towards the claim "The largest prime under 1000 is 997 " would have to be put in a separate category. This strikes me as an unnecessary multiplication of categories, which is why I propose that we run with the more encompassing notion of a transitional attitude. However, see Lord (forthcoming) for some reasons why me might distinguish between suspended judgments that are 'interrogative attitudes' and those that are 'anti-interrogative' attitudes.
} 
regarding who is the murderer. She notices something about his smoking habits, which makes her suspicious of Fred being guilty. At the murder scene, some Camel brand cigarette butts were found. Fred smokes Camels, so this initially seems to incriminate him. However, the butts had filters, whereas Fletcher has only seen Fred smoke filterless Camels. As a result, she becomes very confident that the cigarette butts at the crime scene weren't dropped there by Fred. While this high credence is formed in the process of determining who the murderer is, it is not a transitional attitude in the sense described above. This is because it concludes Fletcher's deliberation about who left the cigarette butts. It terminates her reasoning about what her evidence supports regarding the origin of the cigarettes. For an attitude about some claim $p$ to be transitional in my sense, it can't be formed as a terminal attitude about $p$ that is the result of the agent's deliberation about what her evidence tells her about $p$. To make the point more vivid, notice that agents can of course come to believe all sorts of things while they are in the process of settling a question. Earlier, we went along with Friedman's view that belief is a settling attitude. The beliefs agents form during such an inquiry are not transitional attitudes, and they are not held towards claims that the agent is still deliberating about.

Having laid out and clarified the distinction between transitional and terminal attitudes, I will explain in the next section why this difference matters. I will argue that there are both descriptive and normative differences between terminal and transitional attitudes.

\section{Why the Distinction Matters}

I will begin by pointing out some descriptive differences between transitional and terminal attitudes. Recall that agents form these attitudes during phases of inquiry in which they are evaluating the impact of the evidence they have, rather than during phases in which they collect new information. (As mentioned before, these phases need not be entirely separate, but to simplify the discussion, I look at cases in which they are.) The most obvious descriptive difference is of course the one by which we have distinguished the two types of attitudes in the previous section: terminal, but not transitional attitudes conclude the agent's reasoning about what her evidence supports regarding some question of interest. While terminal attitudes might be followed by further evidence-gathering, and then additional reasoning to incorporate the newly gathered evidence, terminal attitudes are not followed by further reasoning about the evidence the agent already has (unless the agent thinks they might have made a mistake, or they are prone to irrational rumination). By contrast, transitional attitudes are formed in an ongoing reasoning process, and are thus typically followed by further deliberation (unless some interruption occurs that prevents the agent from finishing their deliberation.)

This naturally has consequences for their stability and settledness, as well as their impact on other attitudes. When an agent has finished deliberating about how their evidence bears on some question, the terminal attitude they form as a result of this reasoning usually has a certain amount of stability, and is endorsed by the agent. For the agent to change their mind, they typically have to encounter new relevant information, or they have to somehow become suspicious of the quality of their original reasoning in order to start a new phase of deliberation. Moreover, once an agent has finished deliberating, the conclusion they reach is then typically used to update other 
related attitudes. By contrast, transitional attitudes have a more "throwaway" nature. They serve, in a sense, as stepping stones on the way to the conclusion. They keep track of the results the deliberation has yielded so far, only to be thrown out and overturned the moment the reasoning progresses. Hence, they lack the stability and settledness of terminal attitudes. They also don't seem to be used to update related attitudes in the same way as terminal attitudes, rather, their role is more isolated to the deliberation at hand. ${ }^{5}$

The different roles transitional and terminal attitudes play in reasoning have consequences for how they give rise to actions and assertions. We are generally reluctant to use transitional credences as a basis for assertions and decisions, unless we are forced to do so, for example due to time constraints, or because we are stumped by a reasoning task and don't know how to finish it. In case (4), Una answers the test question based on a transitional credence, but only because the time pressure forces her to do so. While we are still deliberating, we might prefer to give an entirely noncommittal answer to a question, rather than report a transitional credence. Someone who is in the middle of deliberating about a complex question, when asked whether $p$ is the likely answer, might prefer to say "I have no idea" or "I can't tell yet" instead of reporting their transitional credence. Or, if they do report their transitional credence, they are likely to qualify their report to indicate this, for example by saying "It looks like $p$ is the likely answer, but I still need to think more about it." By contrast, terminal credences and suspensions are much more readily used as a basis for assertions and decisions, even when they don't terminate the inquiry.

The differences between terminal and transitional attitudes I have just pointed out were of a descriptive nature, and I submit that they hold at least typically, in agents who are rational. I now want to move on to explain why the difference between these attitudes is normatively relevant. I will argue that our current notions of rationality and justification are far better suited for evaluating terminal attitudes than transitional ones. In order to do so, I will consider both traditional and Bayesian notions of rationality and justification. In traditional epistemology, beginning with Firth (1978), a distinction is standardly made between propositional (or ex ante) and doxastic (or ex post) justification. These notions are commonly spelled out as follows: if an agent has evidence for $p$ (where $p$ could either be a probabilistic or non-probabilistic claim), then the agent is (propositionally) justified to believe $p$ (or have the supported credence in $p$ ). Moreover, for an agent's belief or credence in $p$ to be doxastically justified, their attitude has to be properly based on the evidence that supports it. This means that on the standard way of understanding these notions, doxastic implies propositional justification, but not vice versa. There is some controversy in the literature on how to define these notions, but the details don't matter for my purposes here (see e.g. Turri 2010, Silva 2015, Dogramaci 2018, and references therein for further discussion).

This distinction naturally applies to terminal attitudes. For example, in (1), Fletcher's evidence as she tries to figure out who the murderer is stays constant throughout. Since we've stipulated that she eventually arrives at the justified conclusion that Fred was framed, this claim is propositionally justified throughout her deliberation. It just takes her a while to realize that this is what her evidence supports. Once she figures this out through proper deliberation, her resulting

5 Thanks to Nader Shoaibi for helpful discussion. 
terminal credence and belief are doxastically justified as well. Something similar applies to Una's case. At the outset, she already knows all the relevant logical theorems and principles. Those principles entail that the formula she is considering is tautological, hence this is the propositionally justified conclusion. Once she arrives at this conclusion through proper reasoning, her attitude is doxastically justified as well. What is important to notice here that is that the notion of propositional justification is not relative to an agent's stage in reasoning. Once it has been determined what evidence the agent possesses, this determines which (probabilistic or non-probabilistic) claims are justified for the agent, regardless of whether or how much the agent has reasoned about the claims in question. Different theories of evidence may disagree about how to individuate the evidence that the agent has (by issuing stricter or looser constraints on possession of evidence), but they tend to agree that possessing evidence is independent of whether the evidence has been used in reasoning about a particular question at hand. By contrast, the notion of doxastic justification is sensitive to whether an agent has reasoned well, but due to the standardly assumed entailment between doxastic and propositional justification, it is only sensitive to whether the agent has formed their attitude based on proper reasoning about all the relevant evidence. Hence, neither of these notions is sensitive to the stage of reasoning the agent is currently at.

These consideration demonstrate that the standard way of thinking about justification along the lines of the propositional/doxastic distinction is ill-suited to determine whether transitional credences and suspensions are justified or rational. As I have characterized them, transitional attitudes are formed before and during reasoning, and are usually either only sensitive to part of the agent's evidence, or they consider all of the evidence, but in a way that is less thorough than the agent's terminal, more reflective take on what the evidence supports. For example, Fletcher's initial impression that the evidence probably supports Fred's guilt might very well take into account all of her evidence in some sense, but she hasn't yet noticed subtle inconsistencies that lead her to conclude later that things don't add up in the way they initially appeared.

We can thus rule out any attempt to apply the propositional/doxastic distinction to transitional attitudes in the following way: a transitional attitude is doxastically justified in case it is justified based on the evidence the agent has considered or evaluated at that point. As the Fletcher case shows, an agent might have, in some sense, already considered all of their evidence, but more reasoning about it is needed for the agent to fully appreciate its significance. Hence, this suggestion would wrongly classify Fletchers transitional attitude as unjustified or irrational: if Fletcher has already considered all of her evidence when she initially thinks Fred is probably guilty, this way of evaluating her attitude would force us to say that this attitude is not doxastically justified (because her total evidence supports that Fred is innocent). However, as I've described the case, it seems perfectly fine for Fletcher to be initially impressed by Manny's framing attempt, even though that's not what the evidence really supports. In fact, it would be irrational for her to think Fred is innocent before she has discovered the subtle inconsistencies in the evidence planted by Manny. For this reason, we cannot just straightforwardly apply the doxastic/propositional justification distinction to evaluate transitional doxastic attitudes. We can say the same thing about Una's case. Once she's correctly written out the initial truth table, she has, in a sense, already considered all the relevant 
evidence. However, it still seems rational for her to not adopt an extreme credence until she has double-checked her reasoning.

The same issue arises if we instead try to use a Bayesian conception of rationality to evaluate transitional attitudes. According to the Bayesian notion of rationality, people's degrees of belief should obey the probability axioms, and should be updated by conditionalization. This also entails that logical truths need to be assigned credence 1 . None of these requirements make any reference to how well an agent has deliberated, or what stage of their reasoning process they are at. For this reason, it has recently been argued that Bayesian norms of rationality are best understood as characterizing principles of propositional rationality or justification, i.e. they describe which credences are rational for agents to adopt given their evidence (Staffel forthcoming, Dogramaci 2018, Wedgwood 2017, Titelbaum 2019). According to Bayesian principles, the agents in our examples all have propositionally rational terminal attitudes at the end of their reasoning processes (except Una in (4), whose reasoning is interrupted.) Their attitudes are arguably also doxastically rational, but this is not captured by the standard Bayesian theory. For the same reasons that I laid out above, the standard Bayesian view is not suited to evaluating whether a reasoner has rational transitional attitudes. ${ }^{6}$

As Lisa Miracchi has helpfully suggested to me, some versions of virtue epistemology might provide us with useful resources to explain what makes transitional attitudes rational. Virtue epistemological approaches tend to focus on whether the agent employs appropriate skills or capacities in forming attitudes that promote their epistemic goals, and one might thus focus on whether an agent forms transitional attitudes in a virtuous way. On her knowledge-first virtue epistemology, Miracchi proposes to account for the rationality of suspending judgment as follows: "When an agent generally can be characterized as aiming to A (e.g. aiming to know), we can understand withholding from or omitting a performance of A-ing as manifesting a kind of practical respect for what it takes to A. The agent manifests this respect precisely by not endeavoring to A." (Miracchi forthcoming, p. 26). On this kind of view, we would evaluate transitional attitudes as rational or irrational depending on whether they are formed in a way that respects and/or promotes the epistemic aim. This proposal would of course have to be worked out in more detail, but we can see that the existing framework could be developed in this way.

To sum up our results so far: I have argued that we should distinguish between different roles that non-settling doxastic attitudes, such as suspensions and credences, can play in reasoning. They can either function as transitional or terminal attitudes in reasoning. The notion of a transitional attitude has thus far not been characterized in the literature, but once it comes into focus, it becomes clear that these attitudes play an important role in deliberation. Currently available descriptive and normative characterizations of suspensions and credences seem mostly designed to apply to these attitudes when they play the roles of terminal attitudes in reasoning. Once we home in on these attitudes when they function as transitional attitudes, we see that we

\footnotetext{
${ }^{6}$ In a recent paper on the requirement of logical omniscience, Smithies (2015) explains his views on how Bayesians should account for the rationality of credences held by non-ideal agents. His account strikes me as a promising starting point for Bayesians to develop views on the rationality of transitional attitudes.
} 
face a host of interesting, unanswered questions, some of which I will describe now in the remainder of this section.

On the descriptive side, we face various questions about the nature of transitional attitudes themselves, and also about the nature of reasoning. For example, the way I draw the distinction between transitional and terminal attitudes invites the question: at what point has an agent concluded her deliberation, or evaluation of the evidence? How do we know when they're finished? The best preliminary answer I can give here that the agent has finished their deliberation whenever they have concluded whichever evaluation procedure they are using to determine the impact of the evidence. Sometimes, this evaluation procedure might be carried out as a conscious deliberation, and sometimes it might be carried out at a processing level that is not consciously monitored or accessed by the agent. Of course, not every such procedure will produce a good result. Sometimes agents reason poorly, or make a mistake in implementing an otherwise good reasoning strategy. But either way, both good and bad reasoning processes can deliver conclusions.

Moreover, an agent can in principle use many different strategies for evaluating their evidence, some of which might be less careful, and some might be more careful. For example, a less careful logic student might not double check the result of the truth table with a different method, like Una does. And maybe Una wouldn't do so either if she wasn't completing the work for a grade. For this reason, we can't simply say that a deliberation process is complete once the agent has in some sense taken into consideration all of their evidence. Both Una during the test, and the less careful student have in some sense considered the relevant evidence after doing the truth table, but only the less careful student is done deliberating at this point. Hence, an attitude that one agent considers to be preliminary might be another agent's final answer. The status of an attitude can't be determined based on what evidence has been considered and how it has been considered. It can only be determined in the larger context of the reasoning procedure the agent (or their mind) is carrying out. Thus, an interesting empirical question is how our minds keep track of whether a deliberation is finished or not.

The above considerations also invite further questions about how exactly we should draw the distinction between terminal and transitional attitudes. First, one might wonder whether the distinction is really a sharp one, or whether there could be vague or indeterminate cases. ${ }^{7}$ I am inclined to think that the distinction is not sharp, given the difficulties mentioned above that stem from the question of whether an agent has finished reasoning. Moreover, the evidence collection and evidence evaluation components of inquiry are not always neatly separated, which further complicates a straightforward classification in some cases. Second, we could either construe the terminal and transitional versions of some attitude type as entirely different kinds of attitudes, or we could think of them as the same kind of attitude that can play different roles, and have different characteristics depending on the role they play. Here, the latter option seems more plausible. For example, suppose an agent finishes deliberating by adopting a 0.7 credence in p. A little later, they come to suspect they might have made an error, and so they reopen the deliberation to double check their reasoning. It doesn't seem plausible that the agent adopts an attitude of a different type

7 Thanks to Eddy Chen for raising this question. 
when they reopen the deliberation, rather, they keep the same attitude, but the role it plays changes. ${ }^{8}$

There is also much more to say about how transitional attitudes typically do and don't feature as bases for action, assertion, and belief updating. I claimed above that we're typically far more reluctant to make assertions and decisions based on transitional attitudes than based on terminal attitudes. This much seems right to me, but then again, we don't always have the luxury of carrying out thorough deliberations before we act or speak. I also suggested that terminal attitudes are used to update related beliefs and credences, whereas transitional attitudes are more isolated to the deliberation they participate in. A more careful investigation here is likely to yield interesting insights.

The focus on transitional attitudes also raises interesting issues in normative epistemology regarding our theories of rationality and justification. I argued that transitional attitudes can be rational or irrational - for example, it seems entirely rational for Fletcher to initially respond to Manny's carefully planted evidence by thinking that Fred probably did it. It would in fact be irrational for her not to think this, before she is aware of the subtle inconsistencies that reveal the framing attempt for what it is upon closer inspection. Yet, most currently available theories of justification and rationality are not well suited to deliver this result, since they are designed to apply to terminal attitudes. They are not sensitive to different stages of reasoning and evidence evaluation, as they would have to be in order to give us the right verdicts about transitional attitudes (but see Miracchi forthcoming and Smithies 2015 for theories that could be developed to apply to transitional attitudes). One might also look towards extant accounts of prima facie justification in order to get traction on this question, although I suspect that this won't get us very far, since complex reasoning cases of the kind I am characterizing here are not often discussed in the literature. I am currently working on a theory of justification for transitional attitudes that complements existing theories of justification for terminal attitudes (Staffel, manuscript).

Further work is also needed to respond to potential objections. One might question, for example, whether the notion of a transitional attitude is really necessary, given certain conceptions of what it takes to possess evidence. For example, one might deny that in the cases I have described, the evidence is really constant throughout the deliberation phase. On such a view, reasoning generates evidence that wasn't there before. Thus, one might perhaps say that Fletcher's initial evidence does propositionally support that Fred is guilty (because it makes him look guilty upon superficial inspection), so Fletcher's initial high credence is propositionally and doxastically justified. Her reasoning about the evidence then produces evidence against Fred's guilt, so that at the end of her deliberation, her evidence supports the claim that Fred is innocent, and her low credence in Fred's guilt is propositionally and doxastically justified then. Similarly, we might say about Una that when she produces the truth table, she is generating additional evidence that the formula is a tautology, which she didn't have before. This view - that reasoning generates new evidence - tries to deny that there are transitional attitudes to which the notion of doxastic/propositional justification doesn't apply, by claiming that the evidence the agent has is

8 Thanks to Mason Westfall for raising this question. 
not constant throughout the deliberation phase. In essence, then, this view ultimately denies that the attitudes that I identify as transitional are transitional at all. Rather, using my terminology, they would have to be characterized as terminal attitudes that the agent considers to be supported by the evidence they have at that point. Further deliberation would amount to collecting or generating more evidence.

This view doesn't strike me as very promising. One problem is that the view abandons the very natural distinction between one's evidence, and one's recognition of what the evidence shows. For example, on this view, Fletcher would be wrong if she claimed "Initially, I thought the evidence was incriminating Fred, but then I realized that it didn't really point to Fred after all." Fletcher suggests here that the evidence stays the same, when, on this view, it really doesn't. Rather, her evidence, and what it supported changed, from supporting a high credence that Fred did it to supporting a high credence that he didn't. This characterization is more than just a little strange, given that Fletcher learns no new empirical information about Fred or the murderer during this period. Moreover, this view even more radically implies that at the beginning of her deliberation, Fletcher's evidence really doesn't propositionally justify any answer to the question of who committed the murder (other than perhaps some maximally neutral one), since she has not yet produced any linking evidence through reasoning. This is odd, since we usually think we can determine what someone's evidence supports, even if the person has never thought about how this evidence relates to the claim under consideration. These considerations give a flavor of quite how radically revisionary this view of evidence would have to be in order to abandon the need for postulating transitional attitudes. The view would have to claim that there is never any case in which an agent is deliberating about their evidence, and their current attitudes are not the attitudes that their current evidence supports.

The view that there are transitional attitudes, whose justification needs to be evaluated in a different way than the justification of terminal attitudes, is of course compatible with a more moderate view about the possibility that reasoning can sometimes generate additional evidence or justification (see Balcerak Jackson and Balcerak Jackson 2013. Matheson 2009 can perhaps also be interpreted as having such a view). How exactly we should think of the justification of transitional attitudes ultimately depends on one's more general view towards evidence and justification. Our ultimate answers to these questions have ramifications for all areas of inquiry in which we encounter complex deliberations - for example the law, science, mathematics, and of course also philosophy. And this is just a small sampling of the interesting questions that come into focus once we realize that transitional and terminal attitudes play different roles in our reasoning.

\section{Comparison with other Inquiry-Guiding Attitudes}

In this last section, I will offer a brief comparison between transitional attitudes and other inquiryguiding attitudes that have recently been discussed in the literature. This is to alleviate the potential worry that I am just reinventing the wheel by identifying a kind of inquiry-guiding attitude that has already been characterized elsewhere under a different label. 


\subsection{Acceptance}

The attitude of acceptance has been introduced in epistemology and philosophy of science as an attitude that we might adopt in inquiry. It is usually taken to be importantly different from belief (see Weirich (2004) for an overview of various ways of characterizing acceptance, and also the comparison Fleischer (forthcoming) offers between endorsement and acceptance for further references). Acceptance is often distinguished from belief in that, unlike belief, it is voluntary, and not bound by evidential support. However, like belief, accepted claims are treated as true (at least in some contexts), which involves using them as premises for reasoning and decision-making. For example, I might believe that Newtonian physics is incorrect, but I could still accept it for the purposes of a particular deliberation. It is easy to see that the transitional attitudes I have described are not in any way like acceptances. I've argued that credences and suspensions are paradigmatic transitional attitudes, which, unlike acceptances, don't involve treating anything as true. Moreover, transitional attitudes are unlike acceptances in that they don't seem to be under our voluntary control. They are also sensitive to our evidence (or at least to our current awareness of the significance of our evidence) in a way that acceptance is not. Hence, transitional attitudes are definitely not acceptances.

\subsection{Endorsement}

Fleischer (forthcoming) has recently argued that the attitude of endorsement plays an important role in inquiry. He argues that endorsement "is the appropriate attitude to take toward one's favored theory during the course of inquiry, within the domain of a cutting-edge research field." (p. 4) More specifically, he characterizes endorsement as follows:

Endorsement: Endorsement is a doxastic propositional attitude. $S$ endorses $p$ in a research domain $d$ only if:

1. $S$ is disposed to assert that $p$, or otherwise express commitment to $p$ (in $d$ ).

2. $S$ takes herself to be obligated to defend $p$ (in $d$ ).

3. $S$ treats $p$ as a premise in her further reasoning (in $d$ ).

4. $S$ shapes her research program in $d$ (in part) based on $p$.

5. $S$ is resiliently committed to $p$ (in $d$ ).

6. $S$ takes $p$ to be a live option (i.e. she doesn't know $p$ is false).

7. In endorsing $p, S$ aims to promote healthy inquiry.

Fleischer argues that endorsement is a suitable attitude to take towards a theory one is trying to establish or promote in one's research. It allows commitment to a theory even if one has yet to deliver conclusive evidence that the theory is correct (which one might perhaps never be able to do, just think of how it goes in philosophy...). He goes on to distinguish endorsement from belief and acceptance, and argues for norms that govern rational endorsement.

We need to check whether endorsement is similar to transitional attitudes as I've characterized them. Fortunately, it is easy to see that this is not the case. Given what I've said about the nature of transitional attitudes, they definitely don't meet conditions 1, 2, 3, 4, and 5. Given 
their preliminary nature, agents typically don't express commitment to transitional attitudes or feel the need to defend them (except as appropriate given that they haven't yet finished reasoning), they don't use them as premises in further reasoning, they are not resiliently committed to them, and they don't use them as a basis of their research program (again, unless we count the trivial sense in which we treat an open question as the basis of a research program). In fact, I think endorsing some claim $p$ in Fleischer's sense is compatible with holding some transitional attitudes towards $p$ that can change and evolve in the course of one's deliberation, whereas there might be no change in one's endorsement of $p$ during this time. In sum, the inquiry-guiding attitude of endorsement is not a transitional attitude under a different label.

\subsection{Hypothesis}

Another recently characterized attitude that plays a role in inquiry is Palmira's notion of hypothesis. Palmira is interested in cases of inquiry in which one tries to find the best answer to a given question, for example which theory best explains some biological phenomenon. While one might be initially neutral about which answer is best, Palmira points out that one might at some point start favoring one of the answers, and direct one's inquiry more specifically at confirming (or disconfirming) that this is the answer to the question. He characterizes the attitude one has in this case as follows: "hypothesis is the attitude of being cognitively inclined towards $p$ qua answer to a question" and "one hypothesizes that $p$ only if one treats $p$ as true for the sake of closing one's inquiry into $Q$ via $p . "$ (p.9) Hypothesis is thus distinct from suspension (which is neutral among the answers to $Q$, and also from belief, since a hypothesis of $p$ is can't usually ground assertions and function as a premise in further reasoning, like a belief can. Instead, hypothesizing that $p$ centrally entails focusing one's inquiry on establishing that $p$ is the right answer to $Q{ }^{9}$ Accordingly, hypothesizing that $p$ is appropriate from a normative point of view just in case "entertaining $p$ enables one to make reliable progress with the inquiry into $p$ being the answer to $Q . "$ (p. 14)

Hypothesis is arguably a more tentative attitude than endorsement or acceptance, and so it is perhaps a bit closer to the transitional attitudes I have described. But still, on closer inspection, they are importantly different. Hypothesis is reserved for a given proposition that one takes to be the most plausible answer to a given question, whereas transitional attitudes are held towards all (epistemically) possible answers to the question under consideration. As Palmira himself remarks, this makes suspension and hypothesis distinct. Transitional attitudes merely keep track of the current status of one's deliberation, and transitional credences, in particular, change continuously as one's deliberation evolves. Hypothesis is thus also different in that it is more stable, and doesn't fluctuate during one's inquiry into answering $Q$. Palmira thus rejects the reduction of hypothesis to credences (correctly, in my opinion), though he of course doesn't consider transitional credences specifically, just credences in general.

\footnotetext{
${ }^{9}$ Palmira briefly discusses how hypothesis is different from Fleischer's endorsement on p. 11 of his article. There is also a nice summary of the main differences between suspension, belief and hypothesis on p. 12 that interested readers might want to consult.
} 
In principle we could examine yet more attitudes to ensure they are distinct from transitional attitudes, such as supposition, or conjecture, and I invite the reader to pursue this line of inquiry if they are so inclined. I anticipate that transitional attitudes will not be of a kind with those more familiar doxastic attitudes either. For now, I conclude that, although they are in a sense inquiry-guiding attitudes, they are distinct from acceptance, hypothesis, and endorsement, but could very well be held simultaneously with any of these attitudes.

\section{Conclusion}

I have argued in this paper that we should distinguish between transitional and terminal doxastic attitudes in reasoning. Doxastic attitudes that occur as both of these subtypes include degrees of belief and suspended judgments, whereas outright beliefs - at least on Friedman's view - are only suitable as terminal attitudes. I argued that this distinction is worth drawing because transitional and terminal attitudes differ in their descriptive features, and they are also subject to different criteria of normative evaluation. Specifically, our current standard theories of justification and rationality are well-suited for evaluating terminal attitudes, but not transitional attitudes. Once we understand what transitional attitudes are, it becomes apparent that they play a role in any context in which we encounter complex reasoning tasks. This includes everyday reasoning, but also legal and moral reasoning, philosophical reasoning more generally, scientific reasoning, and mathematical reasoning. Realizing that many of the attitudes we form and reason with in these areas are transitional attitudes helps us better understand the nature of our inquiries, and the justificatory status of the attitudes we form in the process.

\section{Acknowledgements}

I would like to thank Brian Talbot, Lisa Miracchi, and the audience at the $45^{\text {th }}$ Meeting of the Society for Philosophy and Psychology in San Diego in July 2019 for helpful feedback and discussion.

\section{Bibliography}

Balcerak Jackson, Magdalena \& Balcerak Jackson, Brendan. 2013. Reasoning as a Source of Justification. Philosophical Studies 164, 113-126.

Dogramaci, Sinan. 2018. Rational Credence Through Reasoning. Philosophers' Imprint 18 (11), 125.

Firth, Roderick. 1978. Are Epistemic Concepts Reducible to Ethical Concepts? in: A. I. Goldman \& J. Kim (eds), Values and Morals, Dordrecht: D. Reidel, 215-229.

Fleischer, Will. forthcoming. Rational Endorsement. Philosophical Studies.

Friedman, Jane. 2019. Inquiry and Belief. Noûs 53 (2), 296-315. 
Friedman, Jane. 2017. Why Suspend Judging? Noûs 531(2), 302-326.

Friedman, Jane. 2013a. Suspended Judgment. Philosophical Studies 162 (2), 165-181.

Friedman, Jane. 2013b. Rational Agnosticism and Degrees of Belief. Oxford Studies in Epistemology 4, $57-81$.

Lord, Errol. forthcoming. Suspension of Judgment, Rationality's Competition, and the Reach of the Epistemic. in: Sebastian Schmidt and Gerhard Ernst (eds.), The Ethics of Belief and Beyond. Understanding Mental Normativity. Routledge.

Matheson, Jonathan. 2009. Conciliatory Views of Disagreement and Higher-Order Evidence. Episteme 6 (3), 269-279.

Miracchi, Lisa. forthcoming. When Evidence isn’t Enough. Episteme.

Palmira, Michele. 2018. Inquiry and the Doxastic Attitudes. Synthese, online first.

Silva, Paul. 2015. On Doxastic Justification and Properly Basing One’s Beliefs. Erkenntnis 80, 945955.

Smithies, Declan. 2015. Ideal Rationality and Logical Omniscience. Synthese 192 (9), 2769-2793.

Staffel, Julia. forthcoming. Unsettled Thoughts. A Theory of Degrees of Rationality. Oxford: Oxford University Press.

Staffel, Julia. manuscript. Pro Tem Rationality.

Titelbaum, Michael. 2019. Return to Reason. in: Mattias Skipper and Asbjørn Steglich-Petersen (eds.), Higher-Order Evidence. New Essays. Oxford: Oxford University Press.

Turri, John. 2010. On the Relationship between Propositional and Doxastic Justification. Philosophy and Phenomenological Research 80 (2), 312-326.

Wedgwood, Ralph. 2017. The Normativity of Rationality. Oxford: Oxford University Press.

Weirich, Paul. 2004. Belief and Acceptance. in: Ilka Niiniluoto, Matti Sintonen and Jan Wolenski (eds.), Handbook of Epistemology, Dordrecht: Kluwer Academic Publishers, 499-520. 